\title{
Coloboma of inferior eyelid
}

INSERM

\section{Source}

INSERM. (1999). Orphanet: an online rare disease and orphan drug data base. Coloboma of inferior eyelid. ORPHA:155889

Coloboma of inferior eyelid is a rare developmental defect during embryogenesis characterized by a unilateral or bilateral, partial or full-thickness, variably sized defect of the inferior eyelid (ranging from a small notch to complete absence of the entire lid) which is usually triangular in shape (with base at eyelid marg in) and located on the lateral third of the lid. It can occur isolated, associated with facial clefting or as part of a syndrome. 